\title{
Avaliação dos Serviços Públicos de Saúde em Duas Capitais Nordestinas do Brasil
}

\author{
Francisco José Batista de Albuquerque \\ Cynthia de Freitas Melo \\ Universidade Federal da Paraíba
}

\begin{abstract}
RESUMO - Cresce a necessidade de avaliar programas sociais como mecanismo de feedback aos gestores. Objetivou-se, no presente estudo, avaliar os serviços públicos de saúde (Programa Saúde da Família-PSF e Centros de Referência) em duas capitais nordestinas a partir das crenças de seus usuários e daqueles de policlínicas privadas. Os participantes responderam a "Escala de Avaliação do PSF pelos seus usuários". A estrutura organizacional e o atendimento preventivo tiveram avaliação positiva pelos usuários dos três serviços. A infraestrututura, materiais de consumo, atendimento médico e vínculo PSFcomunidade tiveram avaliação positiva apenas pelos usuários da rede pública. Os usuários dos Centros de Referência, embora tenham reclamado da demora no encaminhamento, avaliaram esses centros positivamente. As policlínicas eram preferidas por seus usuários por serem consideradas mais resolutivas.
\end{abstract}

Palavras-chave: avaliação de programas; serviços públicos de saúde; crenças.

\section{Evaluation of the Public Health Services in Two Northeastern Capitals in Brazil}

\begin{abstract}
The necessity to evaluate social programs as a mechanism of feedback to their managers has increased. The present study aimed to evaluate public health services (Family Health Program-PSF and Centers of Reference) in two Northeastern capitals of Brazil based on the beliefs of their users and of those from private polyclinics. Participants answered the "PSF Users Evaluation Scale". The organizational structure and preventive care were positively evaluated by the users of the three services. Infrastructure, consumption materials, medical attendance and PSF-community linkage were positively evaluated only by the public health system users. Although the users of the Centers of Reference complained about the guiding delay, they evaluated those centers positively. The polyclinics were preferred by their users because they were considered more resolute.
\end{abstract}

Keywords: program evaluation; public health services; beliefs.

A assistência à saúde no Brasil pode ser realizada sob os cuidados da rede pública, contemplada no presente estudo a partir do Sistema Único de Saúde (SUS), com ênfase no Programa Saúde da Família (PSF) e nos Centros de Referência; ou a partir de empresas privadas, inseridas neste estudo a partir de policlínicas privadas. Embora constitucionalmente haja o preceito da universalidade de acesso ao serviço de saúde pública (Ministério da Saúde, 2000), na prática, dadas as condições em que são oferecidos, esses serviços se destinam à população de baixa renda, o que, no Brasil, diferentemente de outros países mais desenvolvidos, geralmente significa o oferecimento de serviços de baixa qualidade (Albuquerque, 2002).

A grande lotação dos serviços de saúde deve-se, em parte, à alta demanda de usuários, que gera uma sobrecarga na realização de atendimentos curativos e dificulta/limita a atenção às ações preventivas (Melo, 2007; Oliveira, 2006). Essa problemática se inicia desde a atenção básica, na organização do território de abrangência pelo qual cada Unidade de Saúde da Família (USF) é responsável. Em suas bases teóricas, cada

1 Endereço para correspondência: Centro de Ciências Humanas, Letras e Artes (CCHLA), Sala 47, Universidade Federal da Paraíba, Cidade Universitária. João Pessoa, PB. CEP 58.059-900.E-mail: frajoba@ uol.com.br. equipe de PSF acompanha a população vinculada à sua área adstrita, sendo responsável por, no máximo, 1.000 famílias (Ministério da Saúde, 2001, 2004, 2006). Em contrapartida, é importante salientar que, embora baseado nos programas implantados com sucesso em outros países, como Cuba, Canadá, Suécia e Inglaterra, aqui no Brasil não se segue o padrão ali encontrado de um médico por habitantes, e sim, multiplica-se essa referência trocando a unidade para família, o que implica na multiplicação da proporção de pessoas a serem atendidas por um único PSF. Enquanto Cuba, líder na relação paciente por médico, possui um médico a cada 170 habitantes, e no Canadá esse número sobe para 470, o Brasil, junto ao Chile e Paraguai, ocupa a $61^{\mathrm{a}}$ posição na lista, com 900 habitantes por médico (Doctors of the World, 2007). Provavelmente é aqui onde reside "o ovo da serpente", porque ao multiplicar exponencialmente a demanda, aliada às precárias condições de saúde e de infraestrutura, a qualidade do serviço prestado à população tende a ser insatisfatória. A demanda que vai além da capacidade de atendimento gera um serviço de baixa qualidade e inatingível, ou pouco eficaz.

Essa problemática da sobreposição da capacidade de atendimento, somada a burocracias internas, dificulta também a operacionalização da rede interligada de serviços do SUS e impermeabiliza o acesso do usuário aos demais níveis do sis- 
tema, operacionalizados pelos Centros de Referência (Melo, 2007; Nascimento \& Nascimento, 2005; Oliveira, 2006; Szwarcwald, Mendonça \& Andrade, 2006; Viana, 2006). De acordo com a filosofia dos princípios de hierarquização e integralidade, a equipe do PSF (médico, enfermeiro, auxiliar de enfermagem, agentes comunitários de saúde, odontólogo e auxiliar de consultório dentário) é responsável pelos serviços de complexidade primária, assistindo as pessoas dentro da própria comunidade e minimizando a superlotação nos hospitais. Quando há necessidade, como em casos de pacientes que precisam de atendimento especializado, eles devem ser encaminhados pelos profissionais do PSF para os Centros de Referência de média (nível secundário) e de alta complexidade (nível terciário), sendo necessária uma interligação entre esses níveis de atenção à saúde nos sistemas municipais. Entretanto, na prática, nem sempre o "referenciamento" funciona, e quando feito, aspectos organizacionais dificultam sua eficácia.

Por sua vez, as policlínicas, instituições privadas que crescem no mercado, prestam serviços de médicos especialistas e exames clínicos a baixo custo e com maior resolutividade que os serviços oferecidos pela rede pública de saúde. As policlínicas têm conquistado um público específico da população que, embora seja pobre e não possua planos de saúde, tem condições econômicas que lhe permitem pagar uma consulta médica, por um custo reduzido, evitando o atendimento pelo SUS. Essa realidade foi encontrada nas duas cidades pesquisadas: em "A", o preço da consulta variava entre R\$12,00 e R \$ 35,00, e os exames clínicos custavam entre R\$4,00 e $\mathrm{R} \$ 15,00$; e em "B" estes serviços custavam entre R\$20,00 e R\$ 25,00. Esses valores viabilizam o acesso aos serviços privados de saúde para um público que antes utilizava o SUS por não ter uma segunda opção compatível com suas condições financeiras.

\section{Por que Avaliar Programas?}

À medida que as exigências das experiências anteriores crescem, a avaliação de políticas e programas governamentais assume grande relevância para as funções de planejamento e gestão governamentais. Grupos de pesquisadores, órgãos de controle público (Tribunal de Contas da União-TCU, Tribunal de Contas do Estado-TCE), concorrentes políticos, economistas e mídia exigem, cada vez mais, prestações de contas, não apenas financeiras, mas também dos resultados de suas ações. A população, cada vez mais consciente de sua posição de pagante, e de sujeitos de direitos, reclama, no cotidiano de funcionamento, a resolutividade das ações governamentais. Fiscalização direta que "obriga" a avaliação contínua da eficácia, eficiência e efetividade das políticas e programas.

Nesse cenário, a avaliação de programas sociais surge como ferramenta fundamental para auxiliar, por meio de fee$d b a c k$, nas decisões dos gestores no tocante à implementação, ao processo e aos resultados alcançados pelos seus serviços. Tais informações podem ser utilizadas no aprimoramento de políticas públicas (Belloni \& cols., 2003; Cano, 2004; Silva \& Formigli, 1994; Tanaka \& Melo, 2000).

Para compreender e avaliar programas sociais do governo de um país é imprescindível entender como funcionam as políticas públicas. É necessário ter um olhar voltado para a avaliação do programa sem perder de vista o foco nas questões sociais que ele suscita, pois, pelo menos no Brasil, estes programas surgem das exigências evocadas das necessidades dos grupos sociais menos favorecidos (Melo, 2007).

Albuquerque (2002) explica, todavia, que a percepção dos brasileiros sobre o serviço público é de um serviço destinado para os pobres, que não exige qualidade, diferindo da percepção das pessoas nos países desenvolvidos, em que as instituições públicas cuidam do bem estar social para usufruto de toda a sociedade. Nessa direção, Feliciano (2005) mostra que a principal finalidade da avaliação em saúde é aprimorar a capacidade de oferecer adequada atenção e melhor condição de saúde aos cidadãos. A avaliação exige, portanto, a articulação de uma proposta técnico-política com a prática daqueles que estão executando e usufruindo das ações de saúde, sendo essa prática compreendida e vivenciada de diferentes modos pelos que dela participam. Prega-se, portanto, que a avaliação é, pelo menos teoricamente, uma das etapas de uma política, posterior à sua implementação e destinada a influenciar sua reformulação. Seus resultados são de interesse de todos os atores sociais envolvidos no programa: beneficiários, implementadores, formuladores (Belloni \& cols., 2003; Hartz, 2001; Rico, 2006).

Cozby (2003) defende que a avaliação de programas é uma área importante da pesquisa aplicada, tipo de pesquisa que tem como objetivo examinar questões relativas a problemas práticos e suas soluções potenciais. $\mathrm{O}$ autor sugere ainda que pesquisas de avaliação de programas devem analisar as reformas sociais e inovações introduzidas no governo, em instituições de saúde mental e na assistência a saúde, dentre outras.

De acordo com Lobo (2001), todavia, na prática, o trabalho de avaliação de programas não tem sido feito de forma correta ou com frequência no Brasil, pois não há ainda uma cultura de avaliação sobre gastos públicos, especialmente de programas sociais. As que são feitas têm por base as perspectivas dos economistas, enfatizando dados financeiros. No entanto, um psicólogo seria também indicado para tal tarefa já que o mesmo é o mais preparado profissionalmente para construção de instrumentos de avaliação de comportamento.

Destaca-se, pois, que a tarefa de avaliar programas sociais, tão necessária quanto complexa, também requer um investimento cuidadoso na busca de compatibilização de instrumentos e, sobretudo, de pactuar o objeto da pesquisa e os objetivos da avaliação (Frekete, 2000; Furtado, 2001; Hartz, 2001; Novaes, 2000).

Embora os psicólogos brasileiros demonstrem pouco interesse em relacionar o conhecimento em avaliação de comportamento com avaliação de políticas públicas, eles são conhecedores de conteúdos sobre relações intergrupais, atitudes, diferenças entre gêneros, comportamentos individuais e grupais e, principalmente, sobre crenças, sendo estas aspectos fundamentais da avaliação (Albuquerque, 1999).

Segundo Krüger (2004), as crenças são classificadas como variáveis intervenientes, ou seja, referem-se a fenômenos psicológicos não acessíveis à observação direta; no entanto, podem ser inferidas e integrar hipóteses empíricas, pois são acessíveis à mensuração, ainda que de forma indireta.

Logo, deve-se considerar a importância de se conhecer as crenças dos atores sociais, pois estas se constituem como 
formas de compreensão de sua realidade. O conhecimento das crenças elaboradas pela população a respeito do PSF pode servir de base para a elaboração de estratégias mais eficazes de informação, contribuindo para uma compreensão adequada do funcionamento do PSF, possibilitando uma maior colaboração da comunidade com as estratégias de promoção e prevenção de sua saúde, além da participação no controle social, tal como preconizado nas diretrizes do programa.

O objetivo deste estudo, de caráter descritivo e exploratório, foi, portanto, avaliar um extrato do PSF e dos Centros de Referência em duas capitais nordestinas, as quais foram denominadas de cidade " $A$ " e cidade " $B$ ", a partir das crenças dos usuários de serviços públicos de atenção básica à saúde (PSFs e Centros de Referência) e de policlínicas. Buscou-se, ainda, compreender o motivo de preferência dos usuários de policlínicas pelos serviços de saúde privado. Este estudo consiste em uma tentativa de compreender as condições e os fatores que influenciam a eficácia do atendimento aos usuários nos serviços públicos de saúde e identificar os fatores positivos e negativos do PSF, que possibilitam seu êxito ou o fracasso, informações essas que podem servir como feedback aos gestores públicos, auxiliando no planejamento de estratégias de cuidado e manutenção ou restabelecimento da saúde que sejam efetivas.

Foi considerado importante, ainda, a comparação da realidade de funcionamento do PSF e dos Centros de Referências entre essas duas cidades devido algumas diferenciações que podem ser cruciais na facilitação da acessibilidade e desburocratização do sistema. A primeira diferença é que, apesar de possuírem uma estrutura de PSF semelhante, algumas Unidades de Saúde da Família (USF) da cidade "A" possuem atendimento noturno, fato que pode facilitar o atendimento dos usuários que trabalham ao longo do dia. E uma segunda distinção detecta-se nos sistemas de referência, que são bastante diferenciados. Na cidade "B”, o usuário precisa levar um encaminhamento do PSF à central de marcação para solicitar vaga para exames ou médicos especialistas, não existindo uma rede interligada com os dados dos pacientes. Já em "A", a marcação é feita no próprio PSF, sendo o sistema de registro das USFs do município todo interligado e informatizado. Esse sistema, que se pretende implantar também em "B", provavelmente facilita a rede interligada do PSF com os demais níveis de atendimento do SUS. Desse modo, além de obter informações sobre as crenças que os usuários da rede pública e privada fazem do PSF, pretendeu-se verificar também se essas diferenças operacionais alteram sua avaliação.
Método

\section{Participantes}

A pesquisa foi realizada no primeiro semestre de 2007, em duas capitais nordestinas, dentro de unidades do PSF, Centros de Referência e policlínicas. Foi selecionada uma unidade de atendimento de cada serviço em ambas as cidades. As entrevistas foram realizadas com 180 mulheres adultas, entre 25 e 45 anos, numa amostra não probabilística por conveniência, distribuídas por quota com base dos critérios de cidade ("A" e "B") e serviço utilizado (PSF, Centros de Referência e policlínicas) (ver Tabela 1).

A opção por amostra exclusivamente composta por mulheres foi feita porque, segundo Oliveira (2006), elas compõem a maioria dos usuários do PSF.

\section{Hipóteses}

A presente pesquisa consiste em um estudo correlacional, onde as variáveis antecedentes são os serviços oferecidos pela rede de saúde pública (PSF e Centros de Referência de Saúde) e privada (policlínica) e as variáveis consequentes são as crenças dos usuários sobre o PSF, ou seja, a avaliação do serviço utilizado.

Hipotetiza-se que os usuários do PSF e dos Centros de Referência possuem crenças negativas sobre as condições de atendimento do programa (infraestrutura física, materiais de consumo e estrutura organizacional) e que esses usuários possuem crenças positivas sobre o PSF no que se refere à qualidade do atendimento e ao vínculo entre a Equipe de Saúde da Família (ESF) e a comunidade. Hipotetiza-se, ainda, que os usuários das policlínicas possuam crenças negativas sobre o PSF em todos os aspectos e que preferem o serviço privado por considerarem-no mais resolutivo.

\section{Instrumento}

Baseado no trabalho de Oliveira (2006), foi criada a "Escala de Avaliação do Programa Saúde da Família pelos Usuários". Para a validade de construto, procedeu-se à análise fatorial (com método de estimação Unweighted Least Squares - ULS), com rotação Promax e saturação

Tabela 1. Distribuição amostral segundo os critérios de cidade e de serviços utilizados.

\section{Serviço}

Cidade

\section{PSF}

30

Centros de Referência

30

30

30

30

60

60

180
Policlínicas

TOTAL

60

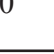

90 
mínima aceitável de $\pm 0,30$, com o objetivo de verificar a dimensionalidade do instrumento de medida. Além disso, os dados foram submetidos à análise da técnica alfa de Cronbach para verificar a precisão da medida (Pasquali, 2003).

A escala, em sua versão final, possui 25 itens de respostas dicotômicas, distribuídos em dois fatores: "condições de atendimento no PSF" (infraestrutura física, materiais de consumo, estrutura organizacional, atendimento médico, vínculo ESFcomunidade) (alpha=0,86), com 16 itens; e "atendimento preventivo e sistema de referência" (alpha $=0,68)$, com nove ítens. Conjuntamente, os dois fatores explicam $28,86 \%$ da variância total e apresentam autovalores (eigenvalues) de 6,14 e 3,45, respectivamente.

Além da escala, seus fatores também foram avaliados por notas atribuídas numa escala de pontuação de variação de 0 a 10 pontos. Foi empregado ainda um questionário biodemográfico (sexo, idade, profissão, renda familiar) para melhor caracterização da amostra, e questões adicionais aos usuários das policlínicas privadas para compreender o motivo de preferências destes por tal serviço.

\section{Procedimento}

A aplicação da escala foi realizada de forma individual nas salas de espera de atendimento das unidades. Considerando-se os aspectos éticos referentes a pesquisas envolvendo seres humanos, os participantes da pesquisas foram informados previamente de que se tratava de um estudo científico e de seus objetivos e procedimentos, do anonimato da sua colaboração, bem como da confidencialidade de suas respostas. Foi solicitado também que os participantes assinassem um Termo de Consentimento Informado, cujo modelo foi elaborado de acordo com a Resolução nº 196/96 sobre Diretrizes e Normas Regulamentadoras de Pesquisa envolvendo Seres Humanos.

\section{Análise de dados}

A análise de dados aconteceu em cinco etapas. Na primeira, foram realizadas analises exploratórias, objetivando limpar o banco de dados, e identificar possíveis erros de digitação. Em seguida, foi realizada uma análise fatorial da "Escala de Avaliação do Programa Saúde da Família pelos Usuários", para verificar a adequação dos itens aos fatores. Na terceira etapa foram utilizadas estatísticas descritivas (frequência, porcentagem, média, desvio padrão) nos dados biodemográficos para fornecer informações acerca da amostra. $\mathrm{Na}$ quarta etapa, foram feitas ainda as frequências e porcentagens de cada item dos fatores. Por fim, foram feitas médias e desvios padrões dos itens das escalas de 10 pontos. Esta foi distribuída em quartil, onde as notas entre 0 e 2,5 foram consideradas como avaliação "insuficiente"; as notas entre 2,5 e 5,0 foram consideradas como avaliação "ruim"; as notas entre 5,0 e 7,5 foram consideradas "boas"; e as notas acima de 7,5 foram consideradas "excelentes".

\section{Resultados}

Como apresentado anteriormente, a presente pesquisa contou com uma amostra composta por usuários de três tipos de serviços: PSF e Centros de Referência, ambos públicos, e policlínicas privadas. Buscou-se verificar a avaliação que esses usuários fazem dos PSFs e Centros de Referência. E, por meio dos usuários das policlínicas, buscou-se verificar ainda as crenças que estes têm sobre o PSF e o motivo de optarem por serviços privados em substituição aos serviços oferecidos pelo SUS, já que é gratuito.

No referente aos usuários das policlínicas privadas, verificou-se que 96,7\% dos entrevistados em ambas as cidades não possuem plano de saúde. Trata-se, entretanto, de pessoas que, mesmo não possuindo seguro de saúde, e com condições financeiras semelhantes às dos usuários da rede pública de saúde, optam pagar pela consulta com preço baixo nas policlínicas. Verificou-se, ainda, que todos os entrevistados nas policlínicas privadas consideram-nas mais eficientes que os serviços da rede pública de saúde. Afirmaram que o atendimento é realizado com maior agilidade, os exames são imediatos e os resultados são entregues em menor tempo. Para eles, isso ocorre porque ao pagarem pelo serviço, este é feito com maior agilidade e com menor burocracia, sem filas de espera, sem pedidos de requisição e sem ter que se deslocar a outros lugares para marcação. Essa burocracia e lentidão seria enfrentada pelos usuários do Sistema Único de Saúde.

Quanto à renda familiar, ao somar os salários e rendimentos de todos os membros da casa, constatou-se que a renda média familiar per capita dos usuários das policlínicas era de $\mathrm{R} \$ 207,47$ pessoa na cidade "B” e R\$ 214,92/pessoa na cidade "A". O valor médio pago pela consulta foi de $\mathrm{R} \$ 31,17$ em "B" e R\$ 25,77 em "A". Isso significa que ao pagar a consulta em "B", o usuário gastou $15,02 \%$ da renda mensal de um individuo da família, e ao pagar a consulta em "A", ele gastou $12 \%$ dessa renda. Ou seja, são indivíduos que gastam uma parcela considerável da sua renda com serviços de saúde que são oferecidos gratuitamente pelo SUS, mas que na prática possuem barreiras que dificultam sua acessibilidade. Isto mostra o quanto um serviço de saúde público eficiente e eficaz contribui com a economia doméstica e, consequentemente, com o bem estar da população, principalmente a de baixa renda.

Buscou-se ainda comparar a renda dos usuários dos três serviços, sob expectativa de que os usuários das policlínicas privadas possuíssem maior renda que os usuários dos serviços de saúde, pois há uma crença disseminada na sociedade de que os pobres vão para o SUS e quem tem melhores condições financeiras recorre aos serviços privados. Ao comparar a renda dos participantes, entretanto, verificou-se que a renda familiar dos usuários das policlínicas $(\mathrm{M}=807,00 ; \mathrm{DP}=509,59)$, dos usuários dos Centros de Referência $(\mathrm{M}=678,10$; $\mathrm{DP}=685,93)$ e dos PSFs $(M=758,00$; $\mathrm{DP}=430,58)$ não apresentam diferença estatisticamente significativa $\left[\mathrm{F}_{(2,19)}=0,83 ; \mathrm{p}>0,05\right]$. Ao lançar, ainda, um olhar mais cuidadoso e analisar essa renda segundo sua quantidade de dependentes, a renda $\mathrm{per}$ capita dos usuários das policlínicas $(\mathrm{M}=223,12 ; \mathrm{DP}=129,52)$ também não diferiu daquela dos usuários dos Centros de Referência $(M=237,57$; $\mathrm{DP}=178,81)$ e do PSF $(\mathrm{M}=227,91$; 
$\mathrm{DP}=132,31),\left[\mathrm{F}_{(2,18)}=0,15 ; \mathrm{p}>0,05\right]$. Ou seja, verificou-se que os sujeitos que optam por pagar pelo serviço da policlínica, ao invés de utilizar os serviços do SUS, possuem renda similar à dos usuários do serviço público. Não se trata, portanto, de sujeitos da classe média, ou mais favorecidos. São pessoas com a mesma faixa socioeconômica que, todavia, optam por um serviço mais eficiente, mesmo que sacrificando parte considerável de sua renda. Contempla-se ainda que os desvios padrões apresentados são altos, pois, além de verificar-se que as rendas dos três grupos são similares, constatou-se também que os grupos não são homogêneos: não há só pobres nos serviços de saúde publica (renda variável entre 150,00 e $4.000,00)$ e não há apenas pessoas de melhores condições financeiras nas policlínicas (mínimo de 200;00 e máximo de $3000,00)$, conforme indicado na Tabela 2.

Após traçado o perfil dos usuários dos três serviços, buscou-se verificar a avaliação que os usuários dos três serviços fazem sobre o PSF. As crenças positivas ou negativas foram identificadas a partir das respostas dadas pelos usuários aos itens da "Escala de Avaliação do Programa Saúde da Família pelos seus Usários", as quais consistiram em notas atribuídas numa escala de 0 a 10 . Vale ressaltar que, como os usuários são escolarizados, foi detectado a posteriori um possível viés devido à tendência de considerarem a nota " 7,0 " como ponto médio por ser esta a "nota" escolar de ponto de corte entre ser aprovado ou não. Portanto, recomenda-se cautela com a possível inflação dos dados tidos como positivos. Feitas essas considerações, a escala foi distribuída em quartil, onde as notas entre 0 e 2,5 foram consideradas como avaliação "insuficiente"; as notas entre 2,5 e 5,0 foram consideradas como avaliação "ruim"; as notas entre 5,0 e 7,5 foram consideradas "boas"; e as notas acima de 7,5 foram consideradas "excelentes".

A Tabela 3 mostra, para o fator "condições de atendimento no PSF", os escores médios atribuídos a cada subfator. Os resultados para as duas cidades sugerem, de modo geral, uma avaliação "boa" da infraestrutura do PSF pelos usuários dos três serviços. Considerando uma escala de 0 a 10 pontos, a média atribuída pelos usuários do PSF foi $6,37(\mathrm{DP}=2,31)$, pelos usuários dos Centros de Referência foi $6,5(\mathrm{DP}=2,43)$, e pelos usuários das policlínicas foi 5,8 $(\mathrm{DP}=1,91)$, não sendo encontrada diferença estatisticamente significativa $\left[\mathrm{F}_{(2,177)}=1,510 ; \mathrm{p}>0,05\right]$ entre essas médias. Observou-se, entretanto, que há uma grande variância nas médias atribuídas. Já as análises gerais das respostas dicotômicas do subfator pelos três tipos de usuários apresentaram frequência de resultados positivos $(50,56 \%)$ e negativos $(49,44 \%)$ semelhantes. Esse resultado indica que, do ponto de vista da estrutura, os serviços não se diferenciam e que, portanto, não é esse o item que mais pesa na decisão dos usuários em preferir pagar pelo serviço nas policlínicas privadas em detrimento ao PSF.

Os materiais de consumo do PSF apresentaram avaliação “excelente" para seus usuários $(\mathrm{M}=7,7 ; \mathrm{DP}=1,85)$ e para os usuários dos Centros de Referência $(\mathrm{M}=7,5 ; \mathrm{DP}=2,02)$, evidenciando que para os usuários da rede pública de saúde é satisfatória a disponibilidade de insumos, distribuição de remédios e contraceptivos. Esses itens receberam avaliação "boa" pelos usuários das policlínicas $(\mathrm{M}=5,6 ; \mathrm{DP}=3,12)$. Foi verificado que existe diferença estatisticamente significativa entre os usuários dos três serviços $\left[\mathrm{F}_{(2,177)}=15,07 ; \mathrm{p}<0,05\right]$. Avaliação positiva também encontrada nas análises dicotômicas, em 61,3\% dos participantes.

Constatou-se, ainda, uma avaliação "boa" da estrutura organizacional de acesso aos serviços do PSF pelos usuários da rede pública. A média atribuída ao PSF pelos seus usuários foi $6,7(\mathrm{DP}=2,32)$ e pelos usuários dos Centros de Referência foi $6,8(\mathrm{DP}=2,2)$. Os usuários das policlínicas, entretanto, avaliaram de forma "ruim" a acessibilidade, com média igual a 4,5 $(\mathrm{DP}=2,32)$. Foi obtida diferença estatisticamente significativa entre os usuários dos três serviços $\left[\mathrm{F}_{(2,177)}=18,67\right.$;

Tabela 2. Renda familiar dos participantes, segundo o serviço utilizado.

\section{Serviço}

Renda

PSF Centros de Referência Policlínicas

$\begin{array}{cccccc}\text { Média } & 758,00(430,58) & \text { Média (DP) } & 678,10 & \text { Média (DP) } & 807,00 \\ \text { (DP) } & & & (685,94) & & (509,59)\end{array}$

Renda familiar

$\begin{array}{lccccc}\text { Mínimo } & 150,00 & \text { Mínimo } & 70,00 & \text { Mínimo } & 200,00 \\ \text { Máximo } & 2400,00 & \text { Máximo } & 4000,00 & \text { Máximo } & 3000,00\end{array}$

Número de dependentes 
Tabela 3. Escores médios atribuídos à infraestrutura, aos materiais de consumo, à estrutura organizacional, ao atendimento médico do PSF e ao vínculo PSF-comunidade pelos usuários do PSF, dos Centros de Referência e das policlínicas privadas.

\section{Usuários dos PSF}

\begin{tabular}{lccc}
\hline Infraestrututura do PSF & $6,37(\mathrm{DP}=2,31)$ & $6,5(\mathrm{DP}=2,43)$ & $5,8(\mathrm{DP}=1,91)$ \\
Materiais de consumo & $7,7(\mathrm{DP}=1,85)$ & $7,5(\mathrm{DP}=2,02)$ & $5,6(\mathrm{DP}=3,12)$ \\
Estrutura organizacional & $6,7(\mathrm{DP}=2,32)$ & $6,8(\mathrm{DP}=2,2)$ & $4,5(\mathrm{DP}=2,32)$ \\
Atendimento médico & $7,7(\mathrm{DP}=1,87)$ & $7,7(\mathrm{DP}=2,09)$ & $5,8(\mathrm{DP}=2,59)$ \\
Vínculo PSF-comunidade & $7,1(\mathrm{DP}=1,94)$ & $7,3(\mathrm{DP}=2,71)$ & $6,6(\mathrm{DP}=2,51)$ \\
\hline
\end{tabular}

p<0,05]. Já nas análises gerais dos três tipos de usuários nas escalas dicotômicas, a estrutura organizacional foi avaliada negativamente por $54,86 \%$ dos participantes.

A avaliação do atendimento médico no PSF apresentouse no quartil superior, com média 7,7 para seus usuários $(\mathrm{DP}=1,87)$ e para os usuários dos Centros de Referência $(\mathrm{DP}=2,09)$. Constatou-se, todavia, uma avaliação "boa" desse subfator pelos usuários das policlínicas $(\mathrm{M}=5,8 ; \mathrm{DP}=2,59)$, verificando-se que existe diferença estatisticamente significativa entre os usuários dos três serviços $\left[\mathrm{F}_{(2,177)}=14,987\right.$; $\mathrm{p}<0,05]$.

Para o vínculo ESF-comunidade, a avaliação dada pelos usuários dos três serviços foi "boa" ou "regular". Constatouse que o nível médio de confiança atribuído à equipe de saúde na cidade "B", numa escala de 0 a 10 , pelos usuários do PSF foi de 7,1 ( $\mathrm{DP}=1,94)$, pelos usuários dos Centros de Referência foi de 7,3 (DP=2,71) e pelos usuários das policlínicas privadas foi de 6,6 $(\mathrm{DP}=2,51)$. Não foi encontrada diferença estatisticamente significativa entre os usuários dos três serviços $\left[\mathrm{F}_{(2,87)}=+0,644 ; \mathrm{p}>0,05\right]$. A média do nível de confiança atribuído à equipe de saúde na cidade " $\mathrm{A}$ " pelos usuários do PSF foi de 8,1 (DP=1,70), dos Centros de Referência foi de $6,8(\mathrm{DP}=2,79)$ e das policlínicas privadas foi de $6,1(\mathrm{DP}=2,68)$. Foi revelada diferença estatisticamente significativa entre os usuários dos três serviços $\left[\mathrm{F}_{(2,87)}=+5,305\right.$; $\mathrm{p} £ 0,05]$. Contempla-se, ainda, que o vínculo também foi avaliado positivamente por $58,06 \%$ dos participantes, na escala dicotômica.

As análises do segundo fator, "atendimento preventivo e sistema de referência", também foram feitas com base nos subfatores. O atendimento preventivo foi avaliado como "bom" pelos usuários do PSF $(\mathrm{M}=5,67 ; \mathrm{DP}=3,74)$ e pelos usuários dos Centros de Referência $(\mathrm{M}=6,00 ; \mathrm{DP}=3,63)$, e foi avaliado como "ruim" pelos usuários das policlínicas $(\mathrm{M}=4,63 ; \mathrm{DP}=3,38)$.

O subfator sistema de referência, apresentou avaliação “excelente", com média igual a 8,33 $(\mathrm{DP}=2,34)$ para eficiência no atendimento e $6,97(\mathrm{DP}=2,56)$ para o encaminhamento. A eficiência no atendimento foi melhor avaliada na cidade "B" $(M=8,83 ; D P=1,21)$ do que na cidade " $A$ " $(M=7,83$; $\mathrm{DP}=3,03)$, sendo essa diferença estatisticamente significativa $(t=1,68 ; \mathrm{p}<0,05)$. Contatou-se, ainda, que o encaminhamento foi melhor avaliado em " $\mathrm{A}$ " $(\mathrm{M}=8,17 ; \mathrm{DP}=2,07)$ do que em
“B” ( $M=7,77 ; \mathrm{DP}=2,99)$, novamente, uma diferença estatisticamente significativa $(t=-0,60 ; \mathrm{p}<0,05)$. Esse resultado indica que o modelo diferenciado de encaminhamento na cidade " $\mathrm{A}$ " resultou em melhor avaliação do sistema de referenciamento.

\section{Discussão}

O Brasil não possui a cultura de avaliação da qualidade dos serviços públicos oferecidos, especialmente os voltados para programas sociais na área de saúde. Esses programas são idealizados e operacionalizados e, muitas vezes, seus resultados não são avaliados. Impera a distância entre os princípios que o regulamentam e aqueles que o gerenciam, e destes com o funcionamento cotidiano do programa junto à sociedade. Como os psicólogos são os profissionais mais capacitados para construírem instrumentos de medidas do comportamento e já que as políticas públicas visam influir na qualidade de vida dos seus cidadãos, cresce a necessidade de que os psicólogos sociais brasileiros demonstrem mais interesse em relacionar o conhecimento em avaliação de comportamento com avaliação de políticas públicas.

O PSF é um desses programas governamentais que envolvem fontes de financiamento, grupos profissionais, usuários e opinião publica, e que necessita da constatação da sua efetiva contribuição na resolução de problemas sociais. É um programa que já possui 15 anos e que seguiu os modelos de assistência às famílias já existentes em Cuba, Canadá, Suécia, Inglaterra e outros países, sendo estruturado em princípios e organização funcional que em sua teoria são, se não ideais, bastante apreciáveis.

Verificou-se uma realidade na prática da atenção à saúde muito adversa ao modelo teórico idealizado, no que se refere aos princípios de universalidade, integralidade, hierarquizção e equidade. Os problemas observados distanciam o SUS teórico, do SUS operacional, aquele encontrado no dia a dia do usuário. Constatou-se no presente estudo, entretanto, que apesar da detecção de queixas, falhas e desvios de condutas dentro das unidades do PSF e dos Centros de Referência, os usuários da rede pública de saúde avaliaram positivamente o PSF em todos os quesitos analisados. Esse resultado revela uma postura de aceitação e aprovação dos serviços públicos que são oferecidos a su- 
jeitos que antes ficavam às margens do acesso aos cuidados em saúde pública.

No presente estudo, as mazelas do sistema foram detectadas pelos usuários das policlínicas privadas. Apesar de terem avaliado positivamente a maioria dos quesitos analisados, eles avaliaram negativamente dois pontos cruciais do programa: o atendimento preventivo e a estrutura organizacional de acesso aos serviços do PSF. A reprovação da quantidade e qualidade do trabalho de prevenção evidencia uma falha no que deveria ser o tripé de trabalho do programa: prevenção, promoção e recuperação de saúde. E a crítica à estrutura organizacional de acesso, representada na quantidade de fichas distribuídas e no tempo de espera nas filas, demonstra barreiras desde o ingresso ao SUS.

Sabe-se que a continuidade da assistência à saúde é dependente da existência de uma rede de serviços de saúde hierarquizada a ser referenciada. Os usuários do presente estudo afirmaram optar pelas policlínicas privadas devido às dificuldades burocráticas presentes no sistema de referência, que consiste no encaminhamento dos pacientes para exames e médicos especialistas do PSF para os Centros de Referência, assim como problemas na contra-referência, que consiste no retorno destes ao médico solicitante. Esse problema existe devido à má integração do PSF com o restante da rede pública de saúde, que resulta em filas de espera que podem prolongar-se por meses. Esse fato contribui para a privatização da saúde, haja vista o aumento das policlínicas dirigidas à população "pobre".

Os resultados aqui obtidos corroboram a ideia de que um dos maiores problemas no contexto brasileiro da saúde é a tendência a ver a saúde pública como uma prática destinada aos pobres, como se não fossem merecedores de coisa melhor. Já é conhecimento de todos que a classe média brasileira optou por pagar os planos de saúde privados, deixando à população menos privilegiada o atendimento público, de baixa qualidade. $\mathrm{O}$ presente estudo mostra que, agora, não apenas a classe média alta, mas também as classes baixas, com igual renda à dos usuários do SUS, e que possuem condições de pagar pelos serviços das policlínicas privadas, têm se esquivado da rede pública de saúde.

Os dados surpreenderam ainda ao evidenciarem que os usuários dos serviços de saúde formam um público heterogêneo em seu nível de renda. Não há somente pobres no serviço público. E não há apenas pessoas com melhores condições financeiras nas policlínicas privadas. Além disso, os usuários das policlínicas apresentam renda média similar aos usuários dos serviços públicos. A grande diferença entre eles não é o nível socioeconômico. É a avaliação que fazem sobre o PSF que os distinguem. São sujeitos que corrompem o conformismo do "é melhor que nada". São sujeitos que recorrem às policlínicas por a considerarem mais resolutiva. Opção essa que custa-lhes, por consulta ou exame, em média, $15 \%$ da renda per capta da família. Esse fato diferencia nossa população da cubana, canadense e européia, que utilizam em massa os serviços públicos gratuitos de saúde. Deve-se considerar que a própria existência das policlínicas privadas e sua crescente demanda evidenciam a necessidade de um serviço público mais resolutivo e que ofereça melhores condições de acolhimento aos seus usuários.
Verificou-se, também, a avaliação positiva dos usuários dos Centros de Referência no que se refere ao encaminhamento do PSF aos níveis de atenção mais complexos e à sua resolutividade. São sujeitos que reconhecem e protestam a demora no encaminhamento de determinadas especialidades, mas que recorrem aos serviços públicos de saúde por falta de alternativas. Ainda, embora a eficácia do atendimento tenha sido melhor avaliada na cidade "B" do que na cidade "A"; observou-se que o encaminhamento foi melhor avaliado em "A" do que em "B". Esses dados mostram que o diferencial apresentado na cidade "A", onde há o controle de marcação de consultas feitas no próprio PSF, com sistema de registro dos pacientes de diferentes USFs do município todo interligado e informatizado, contribui para a qualidade e agilidade do encaminhamento do usuário do PSF ao Centro de Referência. Isso deveria servir de exemplo às demais capitais brasileiras.

Apesar dos avanços com a Constituição de 1988, que definiu a saúde como direito humano básico, e com a criação do SUS, que possui princípios de promoção de saúde, tais como universalidade, integralidade e equidade na busca de uma melhoria da qualidade de vida da população, uma análise da realidade demonstra que o Brasil ainda se apresenta dividido por setores fragmentados em suas ações, desvinculando o social do econômico.

Muitos tentam modificar o modelo de saúde brasileiro, o criticam, e defendem a mudança das estruturas do PSF. Ao término deste trabalho, propõem-se, entretanto, que o problema não seja procurado nos papéis e nas formulações teóricas do modelo, e sim na operacionalização, no cotidiano do repasse de verbas, no trabalho das equipes de saúde e no atendimento aos usuários. Trata-se de mitigar as barreiras que existem entre os dois extremos do programa, aproximando a teoria e a prática. Trata-se de ter em mãos um programa ideal, mas que necessita da operacionalização concreta de suas formulações, que devem sair da utopia e entrar na realidade do atendimento. Um programa que precisa de constante avaliação junto aos seus usuários, que até então têm sido tratados como cobaias conformadas de um constante "é melhor do que nada".

Sabe-se que a temática da saúde apresenta-se ampla e camuflada por ideais que não revelam sua realidade. Todavia, espera-se que os resultados apresentados possam contribuir para a literatura sobre o tema da saúde pública, e mais especificamente para a avaliação do PSF, além de servir para a expansão do debate científico sobre a operacionalização de políticas públicas destinadas a essa demanda, instigando os psicólogos brasileiros a se interessarem por essa temática.

É preciso combater o mito existente no Brasil de que os serviços públicos são destinados apenas à população pobre, que fazem uso de um serviço precário, por esse ser a única alternativa. É necessário que se busque uma real universalidade no atendimento dos PSFs. E que a equidade realmente leve saúde a quem mais precisa, porém, com qualidade e dignidade. É condição sine qua non para um bom funcionamento do SUS, a real participação da população na gestão dos PSFs e a quebra das barreiras de integração entre os diferentes níveis do SUS, através da criação de uma rede integrada e informatizada de dados, e da desburocratização da marcação de consulta. 
Por fim, espera-se que o presente trabalho seja utilizado como um mecanismo de feedback, aproximando os gestores do programa e seus usuários, os ideais preconizados e a realidade de funcionamento. Não se pretende propor novas formas de funcionamento do SUS, mas, sim, identificar as falhas que impossibilitam o funcionamento do PSF tal como preconizado.

\section{Referências}

Albuquerque, F. J. B. de (1999). Apontamentos para uma Psicologia Sociorrural no Brasil [Resumo]. Em Universidade Federal da Paraiba (Org.), Anais do I Congresso Norte e Nordeste de Psicologia (pp. 12-17). Salvador: NEHP/CPD/UFBA.

Albuquerque, F. J. B. de (2002). Social psychology and rural life in Brazil. Psicologia: Teoria e Pesquisa, 18, 37-42.

Belloni, I., Magalhães, H., \& Sousa, L. C. (2003). Metodologia de avaliação em politicas públicas. Coleção Questões da Nossa Época (3 $3^{\text {a }}$. ed.). São Paulo: Cortez.

Ministério da Saúde (2000). Sistema Único de Saúde (SUS): princípios e conquistas. Brasília: Ministério da Saúde.

Ministério da Saúde (2001). Guia prático do Programa Saúde da Familia. A saúde bucal faz parte da saúde da família? Retirado em 21/07/2010, de http://dtr2002.saude.gov.br/caadab/ arquivos $\% 5$ Cguia psf1.pdf.

Ministério da Saúde (2004). Atenção básica e a saúde da família. Retirado em 18/06/2006, de http://dtr2004.saude.gov.br/ dab/atencaobasica.php.

Ministério da Saúde (2006). Política nacional de atenção básica. Série A. Normas e manuais étnicos. Série Pactos pela Vida, Vol. 4. Brasília: Ministério da Saúde.

Cano, I. (2004). Introdução à avaliação de programas sociais. Rio de Janeiro: FGV Editora.

Cozby, P. C. (2003). Métodos de pesquisa em ciências do comportamento. São Paulo: Atlas.

Doctors of the World (2007). Number of inhabitants per doctor. Retirado em 14/08/2008, de http://adsoftheworld.com/media/print/ doctors_of_the_world_netherlands_perspective?size=_original.

Feliciano, K. V. O. (2005). A relação entre o avaliador e o objeto avaliado. Revista Brasileira de Saúde Materno-Infantil, 5, 83-92.

Frekete, M. C. (2000). Estudo da acessibilidade na avaliação dos serviços de saúde. Manuscrito não publicado, Projeto GERUS, Universidade Federal de Minas Gerais, Belo Horizonte.

Furtado, J. P. (2001). Um método construtivista para a avaliação em saúde. Ciência e Saúde Coletiva, 6, 165-181.

Hartz, Z. M. A. (2001). Avaliação e gestão municipal de saúde. Revista Saúde em Foco/Informe Epidemiológico em Saúde Coletiva, 21, 7-19.
Krunger, H. (2004). Crenças compartilhadas, preconceitos e discriminações [Resumo]. Em Sociedade Brasileira de Psicologia (Org.), Resumos de comunicações científicas, XXXIV Reunião Anual da Sociedade Brasileira de Psicologia. Ribeirão Preto: SBP.

Lobo, T. (2001). Avaliação de processos e impactos em programas sociais - algumas questões para reflexão. Em E. M. Rico (Org.), Avaliação de políticas sociais: uma questão em debate (pp. 29-39) (3a. ed.). São Paulo: Cortez.

Melo, C.F. (2007). Avaliação do Programa Saúde da Família (PSF) em João Pessoa e Fortaleza a partir das crenças das usuárias dos PSFs, dos Centros de Referências e das Policlínicas. Monografia de graduação, Universidade Federal da Paraíba, João Pessoa.

Nascimento, M. S., \& Nascimento, M. A. A. (2005). Prática da enfermeira no Programa de Saúde da Família: a interface da vigilância da saúde versus as ações programáticas em saúde. Ciência e Saúde Coletiva, 10, 333-345.

Novaes, H. M. D. (2000). Avaliação de programas, serviços e tecnologias em saúde. Revista Saúde Pública, 34, 547-559.

Oliveira, S. F. (2006). Avaliação do Programa Saúde da Família: Uma análise a partir das crenças dos profissionais da equipe de saúde e da população assistida. Dissertação de Mestrado, Universidade Federal da Paraíba, João Pessoa.

Pasquali, L. (2003). Psicometria: teoria dos testes na Psicologia e Educação. Petrópolis:Vozes.

Rico, E. M. (2006). Avaliação de políticas públicas: uma questão em debate (4a. ed.). São Paulo: Cortez.

Silva, L. M. V., \& Formigli, V. L. A. (1994). Avaliação em saúde: limites e perspectivas. Caderno de Saúde Pública, 10, 80-91.

Szwarcwald, C. L., Mendonça, M. H. M., \& Andrade, C. L. T. (2006). Indicadores de atenção básica em quatro municípios do Estado do Rio de Janeiro, 2005: resultados de inquérito domiciliar de base populacional. Ciência e Saúde Coletiva, 11, 643-655.

Tanaka, O. Y., \& Melo, C. (2000). Uma proposta de abordagem transdisciplinar para avaliação em Saúde. Interface - Comunicação, Saúde, Educação, 7, 113-118.

Viana, A. L. (2006). Modelos de atenção básica nos grandes municípios paulistas: efetividade, eficácia, sustentabilidade e governabilidade. Ciência e Saúde Coletiva, 11, 577-606.

Recebido em 16.02.2009

Primeira decisão editorial em 29.06.2009

Versão final em 24.08.2009

Aceito em 30.09.2009 